\title{
Heat pulse line-source method to determine thermal conductivity of consolidated rocks
}

\author{
M. Fernandez and E. Banda \\ Senei Geologic de Catalunya, Travessera de Gracia 56, 08006 Barcelona, Spain \\ E. Rojas
}

Departament de Termologia, Facultat de Fisiques, Universitat de Barcelona, Avda. Diagonal 645, 08028 Barcelona, Spain

(Received 30 January 1986; accepted for publication 18 July 1986)

\begin{abstract}
An instrument designed to measure thermal conductivity of consolidated rocks, dry or saturated, using a transient method is presented. The instrument measures relative values of the thermal conductivity, and it needs calibration to obtain absolute values. The device can be used as heat pulse line source and as continuous heat line source. Two parameters to determine thermal conductivity are proposed: TMAX, in heat pulse line source, and SLOPE, in continuous heat line source. Its performance is better, and the operation simpler, in heat pulse line-source mode with a measuring time of $170 \mathrm{~s}$ and a reproducibility better than $2.5 \%$. The sample preparation is very simple on both modes. The performance has been tested with a set of ten rocks with thermal conductivity values between 1.4 and $5.2 \mathrm{~W} \mathrm{~m}^{-1} \mathrm{~K}^{-1}$ which covers the usual range for consolidated rocks.
\end{abstract}

\section{INTRODUCTION}

Thermal conductivity of rocks is an important parameter in earth's heat flow determination, engineering applications, and geological and geophysical exploration. Measuring thermal conductivity is performed using either steady-state or transient regime. The most common among the steady-state methods uses a divider-bar apparatus. The main difficulties of the divided-bar method are the time involved in each measurement (10-60 min depending on the design ) and the sample preparation (disks of different thicknesses with flat and parallel surfaces). Instruments based on transient methods improve the measuring time (2-10 $\mathrm{min}$ ), and generally, the sample preparation is easier.

The most popular of the transient methods is the "needle probe method" "which uses a continuous heat line source in a homogeneous, isotropic, and infinite medium. Other transient methods ${ }^{2,3}$ use a heat pulse line source. These methods present a cylindrical symmetry and are especially suitable for measurements in unconsolidated sedimentary rocks. However, their use in measuring consolidated rocks faces severe difficulties, in particular, thermal contact resistance, reproducibility, and sample preparation.

Systems based on the solution of the heat conduction equation for a flat plate insulated thermally on one side and heated on the other side ${ }^{4.5}$ have been developed to measure thermal conductivity, thermal diffusivity, and heat capacity of consolidated rocks.

A recent design commercially available (QTM-2 from Showa Denko) is based on a transient method using a continuous line source. ${ }^{6}$ The design presents a half-cylinder symmetry where the temperature variation, for a given time period, is similar to that of the needle probe method.

In this paper a system to measure thermal conductivity of consolidated rocks, similar to the QTM-2, is presented.
The new instrument includes important variations with respect to the QTM-2 both in the design and parameters used to derive the thermal conductivity. This instrument is significantly cheaper than instruments commercially available and can be built very simply in any laboratory. The system can be used either in the heat-pulse-source mode or in the continuous heat source mode. An analysis of the results obtained using both modes is presented and compared with conductivities determined with a divided-bar device.

\section{THEORY}

We can assume, for simplicity, a theoretical model based on an infinite line source in a homogeneous, isotropic, and infinite medium. The differential equation to be solved is

$$
\frac{\partial T}{\partial t}=a^{2} \frac{\partial^{2} T}{\partial \mathbf{x}^{2}}+\delta(\mathbf{x}, t)
$$

with $T(\mathbf{x}, 0)=T_{0}$ and $T(\infty, t)=T_{0}$, where $\mathrm{x}=(x, y, z), a^{2}$ is the diffusivity thermal coefficient, and $T_{0}$ is the initial temperature. If we solve Eq. (1) for a continuous heat source, the result is ${ }^{7}$

$$
T\left(r_{0}, t\right)=(Q / 4 \pi k) \operatorname{Ie}\left(r_{0}^{2} / 4 a^{2} t\right)+T_{0},
$$

where $k$ is the thermal conductivity, $l e$ is the exponential integral, $r_{0}$ is the distance to the heat source, and $Q$ is the heat for unit length and time given by the heat source. $T\left(r_{0}, t\right)$ can be simplified for a large time $(t>30 \mathrm{~s})$,

$$
T_{2}-T_{1}=(Q / 4 \pi k) \ln \left(t_{2} / t_{1}\right),
$$

where $T_{2}$ and $T_{1}$ are the temperatures at $t_{2}$ and $t_{1}$, respectively.

For a heat pulse source acting during a time interval $0<t<A$, where a rectangular heat pulse is assumed, the solution of (1) is (2) for $0<t<A$. 


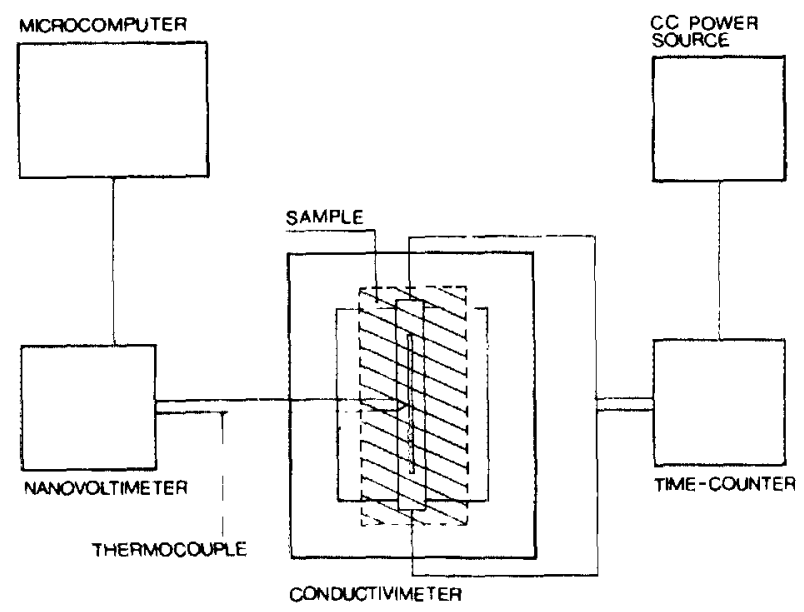

F1G. 1. General scheme of the elements of the experimental apparatus.

For $t>A$ the process is that of a free redistribution of temperatures with the condition

$$
T\left(r_{0}, A\right)=(Q / 4 \pi k) \operatorname{Ie}\left(r_{0}^{2} / 4 a^{2} A\right)+T_{0},
$$

the solution being

$$
\begin{aligned}
T\left(r_{0}, t\right)= & T_{0}+\left[Q / 16 \pi^{2} a^{2}(t-A)\right] \\
& \times \int_{\mathbf{R}^{2}} I e\left(\frac{r_{0}^{2}}{4 a^{2} A}\right) \exp \left(\frac{-\left(r_{0}-r\right)}{4 a^{2}(t-A)}\right) d^{2} \mathbf{r},
\end{aligned}
$$

where $\mathbf{r}=(x, y)$.

\section{EXPERIMENTAL APPARATUS}

The experimental apparatus is made up of the following elements: heating system (heater, time counter, and power source), temperature measuring system (thermocouple, nanovoltmeter, and microcomputer), and supporting frames (Fig. 1).

The heater consists of an electric resistance coil placed inside an aluminum parallelepiped bar $\left(62 \times 3 \times 4.5 \mathrm{~mm}^{3}\right)$. The aluminum bar is inserted in a slot of the polyvinyl chloride (PVC) frame in such a way that it remains thermally isolated by an air-filled space. The heater is connected with a time counter which can operate in time intervals between 1 and $999 \mathrm{~s}$ with a resolution better than $100 \mu \mathrm{s}$. In turn, the time counter is connected to a precision power source (HP 6114-A) of $1-\mathrm{mV}$ sensitivity and a stability better than $0.025 \%$.

The temperature measuring system is a chromel-constantan thermocouple whose sensor is inserted in a small

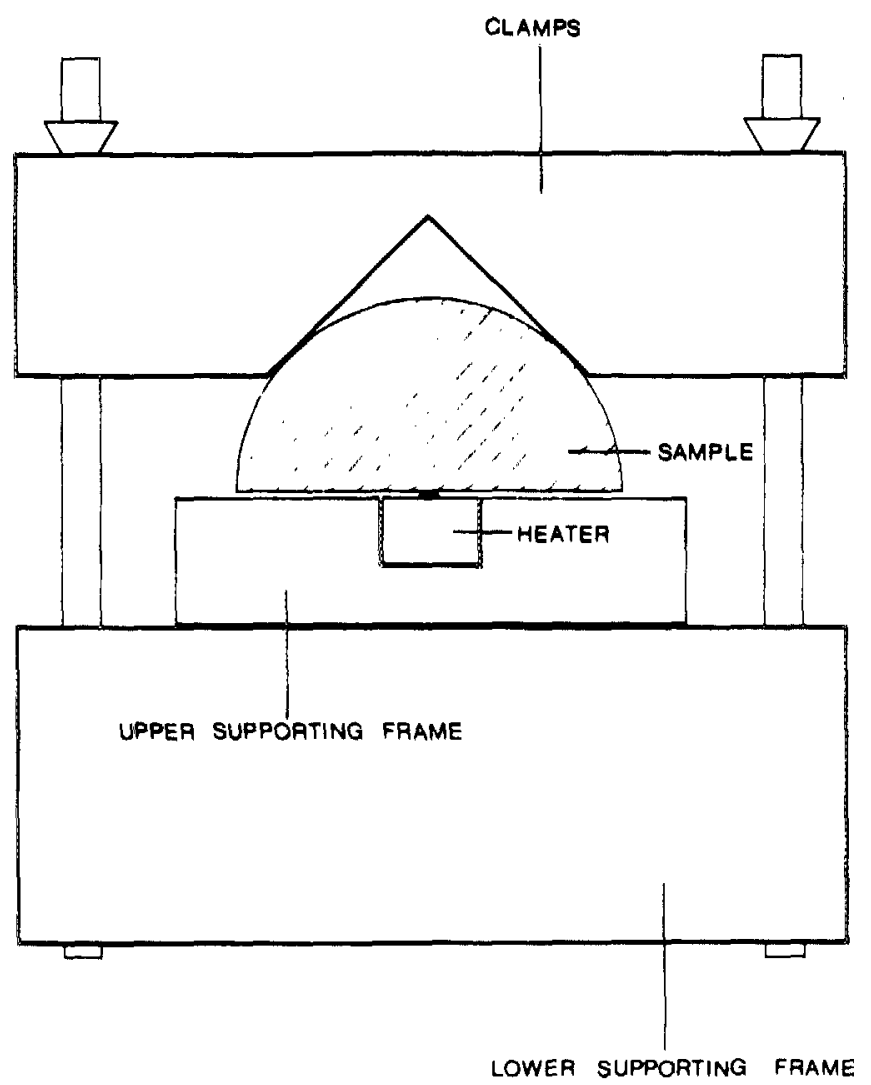

FIG. 3. Front view of the thermoconductometer.

hole in the center of the aluminum bar. The voltage generated by the thermocouple is measured with a nanovoltmeter (Keithley-181) of 61-digits amplitude scale and $10-\mathrm{nV}$ resolution, connected to a microcomputer via IEEE-488 bus, which stores and processes the data.

The heater and the thermocouple sensor are inserted in the upper supporting frame with a balancing system which allows the mechanical-thermal coupling between the heater and the rock samples. This set is mounted on the lower supporting frame which has two clamps to hold the sample tightly over the heater (Figs. 2 and 3 ).

The sample preparation is extremely simple, requiring a roughly flat surface of about $50 \times 80 \mathrm{~mm}^{2}$. The heater configuration allows us to measure indistinctly dry or wet samples. Once the sample has been placed on the heater the temperature variation with time can readily be measured.

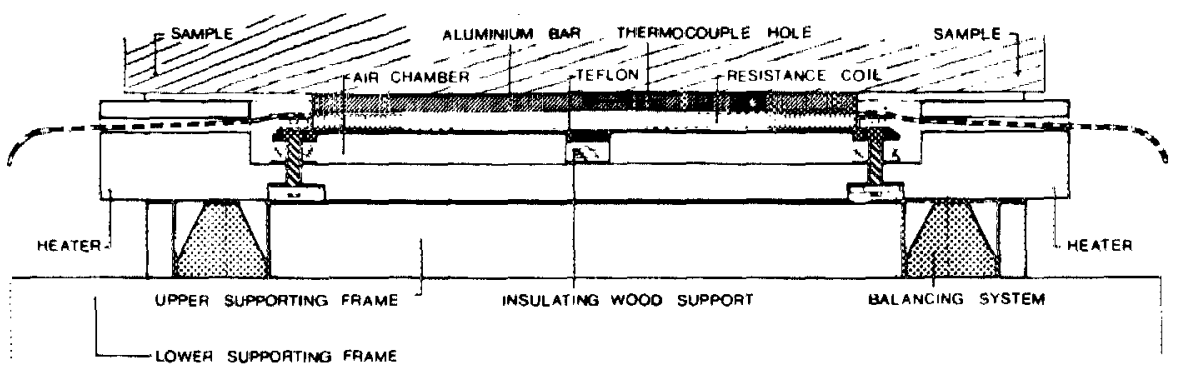

Fic. 2. Longitudinal section of the heater and supporting frame. 


\section{HI. WUSTRUMENT RESPONSE}

The solution of Eq. (1) given previously is not directly applicable to our design because of deviation of the experimental design with respect to the theoretical model. Therefore, the following effects will have to be taken into account: half-cylinder symmetry and heat diffusion through the lateral and lower sides of the heater, finite-section and finitelength of the heater, sample finiteness, and thermal contact resistance.

In order to qualitatively evaluate these effects a typical curve of instrument response, or thermal evolution curve during 3000 s, has been obtained (Fig. 4). According to the theoretical model, this curve should be a straight line ( $T \simeq A \ln B t$, where $A$ and $B$ are constants). The deviation can be described by a sequence of time intervals in which the dominant effects are interpreted as follows:

During the first few seconds, zone A ( 0 to $3-4 \mathrm{~s}$ ), the temperature increases very slowly because of the thermal inertia of the heater.

Within the next interval, zone $B(4-11 \mathrm{~s})$, an abrupt change can be observed, and the temperature rises quickly because heat loss through the lateral and lower sides of the aluminum bar is prevented by the air chamber.

Zone C (11-23 s) is characterized by heat loss through the air chamber. There is no thermal inertia effect and the influence of the sample is not yet felt.

In zone $\mathrm{D}(23-100 \mathrm{~s})$ the temperature increase slows down because the rock sample starts to influence the temperature pattern.

Zone E $(100-400 \mathrm{~s})$ is characterized by logarithmic temperature variation with time and is interpreted as the interval where the rock sample influence is dominant.

In zones $F$ and $G(400-1500 \mathrm{~s}$ and $1500-3000 \mathrm{~s}$, respectively) the heat loss through the sample is responsible for a new change in the variation of temperature with time. On the other hand, the finite length of the line source is

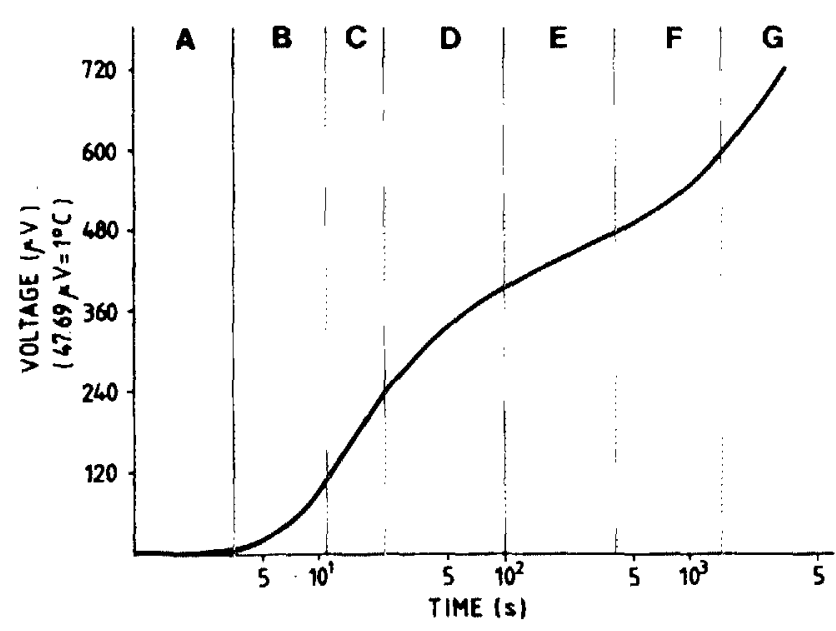

FIG. 4. Instrument response curve. Dominant effect in zone $A$, finite section of the heater (thermal inertia); B, thermal inertia and heat loss through the heater; $C$, heat loss through the heater; $D$, heat loss through the heater and conduction through the sample; $E$, conduction through the sample; F, conduction through the sample and heat loss; $G$, heat loss through the sample. responsible for a certain influence of the medium thermal diffusivity in the measurement of the thermal conductivity $(k)$ following the expression

$T\left(r_{0}, t\right)=\left(\frac{Q}{4 \pi k}\right) \int_{-l / 2}^{l / 2}\left(\frac{1}{\left(r_{0}^{2}+z^{2}\right)^{1 / 2}}\right) \operatorname{erfc}\left(\frac{r_{0}^{2}+z^{2}}{4 a^{2} t}\right)^{1 / 2} d z$,

where $\operatorname{erfc}(x)$ is the complementary error function and $l$ is the heat source length.

Therefore, the thermal conductivity determination is influenced by the thermal diffusivity of the sample itself, giving some uncertainty to the measurement. The uncertainty is time dependent according to expression (6) whose numerical evaluation for extreme values of the diffusivity $(0.005$ and $0.02 \mathrm{~cm}^{2} / \mathrm{s}$ ) gives the following results. Time: 60,300 , and $500 \mathrm{~s}$; maximum error: $0.08 \%, 4.5 \%$, and $6.2 \%$, respectively. Furthermore, a thermal contact resistance will give a higher value for the measured temperature. However, only the reproducibility of the thermal contact resistance will affect our results. For this reason, a flat surface for the heating device has been designed.

Obviously, the different effects influence the whole process, but their relative relevance is a function of time. We can, therefore, consider that the dominant effect in region $\mathrm{E}$ (Fig. 4) is the heat conduction through the sample. In this region (300-s time interval) the temperature increases roughly as a direct function of the logarithm of the time, although it stricly represents an inflection zone. Usually this zone is associated with a straight line whose slope is used to determine the thermal conductivity. However, delimiting the extremes of zone $\mathrm{E}$ can lead to significant errors. For this reason a new measuring parameter is proposed which is based on delimiting the region $E$ in a rough manner and adjust the set of points to a polynomial of seventh degree which can be fitted with a precision better than $0.03 \%$. Subsequently, the inflection point and its slope can be computed. In this way we prevent the use of subjective criteria in determining the slope. Therefore, our parameter for thermal conductivity determination is the slope of the inflection point (SLOPE).

On the other hand, it is evident that with the theoretical model used here measuring the slope is equivalent to determining the temperature at a given time. The easiest way to do that, in practice, is to use a heat pulse source and measure the

TABLE I. Thermal conductivity, TMAX, and SLOPE parameters obtained from reference samples.

\begin{tabular}{lccc}
\hline Sample & $\begin{array}{c}\text { Conductivity } \\
\left(\mathrm{W} \mathrm{m}^{-1} \mathrm{~K}^{-1}\right)\end{array}$ & $\begin{array}{c}\text { TMAX } \\
(\mu \mathrm{V})\end{array}$ & $\begin{array}{c}\text { SLOPE } \\
\left(\mu \mathrm{V}^{-1} \times 10^{2}\right)\end{array}$ \\
\hline Massive gypsum & $1.44 \pm 0.18$ & 216.75 & 9.46 \\
Basalt & $2.22 \pm 0.16$ & 170.45 & 13.70 \\
Quartzdiorite & $2.54 \pm 0.03$ & 151.60 & 14.54 \\
Granodiorite & $2.86 \pm 0.17$ & 135.42 & 16.31 \\
Grainstone & $2.92 \pm 0.03$ & 133.23 & 17.61 \\
Granite & $3.28 \pm 0.03$ & 110.22 & 20.92 \\
Dolomite & $3.34 \pm 0.15$ & 120.76 & 19.17 \\
Sandstone & $4.39 \pm 0.28$ & 108.66 & 20.09 \\
Microconglomerate & $4.49 \pm 0.33$ & 100.04 & 23.40 \\
Marble & $5.19 \pm 0.07$ & 87.70 & 25.45 \\
\hline
\end{tabular}




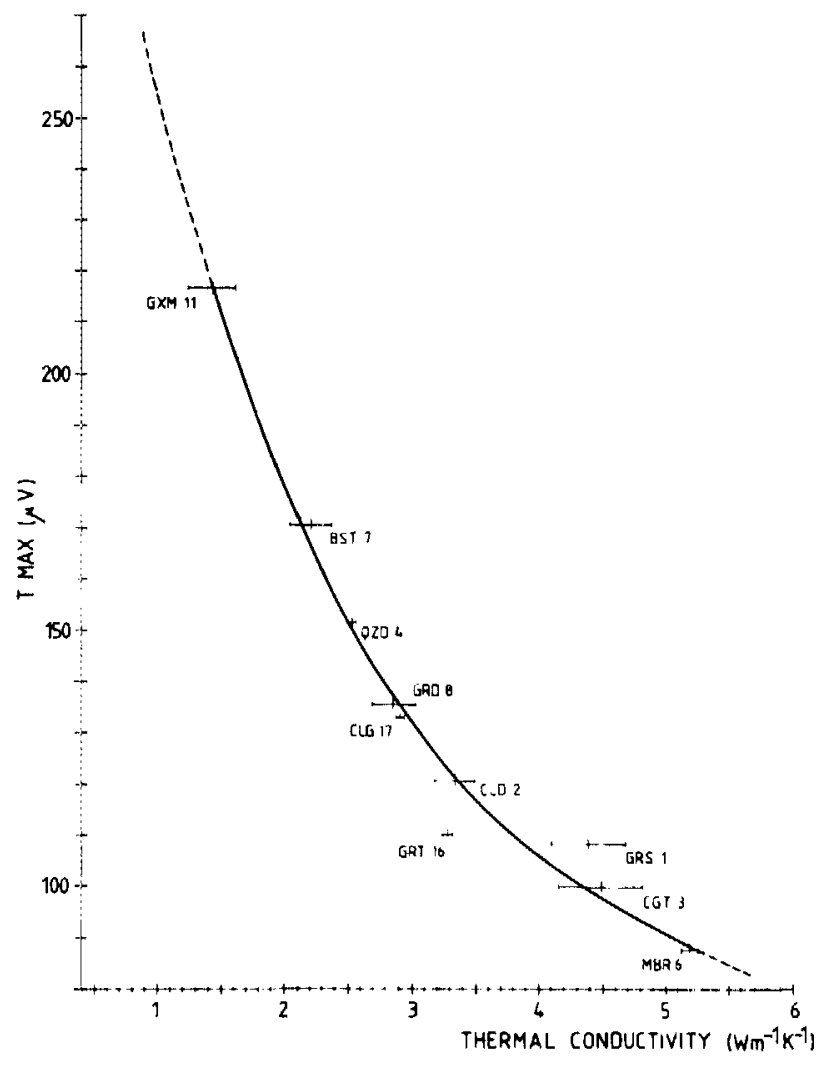

Fig. 5. Graphic representation of the calibration function in heat-pulsesource mode with corresponding error bars. Codes: GXM11, massive gypsum; BST7, basalt; QZD4, quartzdiorite; GRD8, granodiorite; CLG17, grainstone; CLD2, dolomite; GRT16, granite; GRS1, sandstone; CGT3 microconglomerate; MBR6, marble.

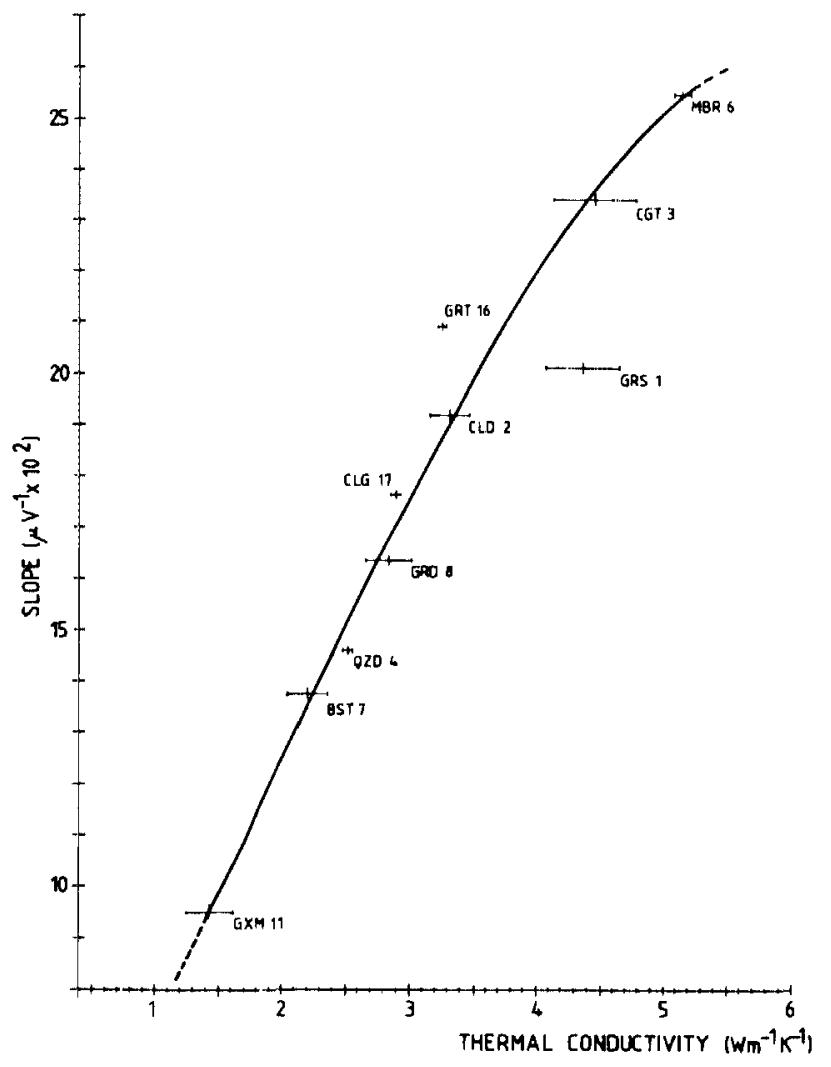

FiG. 6. Graphic representation of the calibration function in continuous heat source mode with corresponding error bars. Sample codes as in Fig. 5.
TABLE II. Reproducibility of the conductometer in the heat-pulse-source mode.

\begin{tabular}{lccc}
\hline \hline Sample & $\begin{array}{c}\text { TMAX } \\
(\mu \mathrm{V})\end{array}$ & $\begin{array}{c}\text { conductivity } \\
\left(\mathrm{W} \mathrm{m}^{-1} \mathrm{k}^{-1}\right)\end{array}$ & Reproducibility \\
\hline Granodiorite & 129.75 & 3.06 & \\
Granodiorite & 130.39 & 3.06 & $\pm 2.5 \%$ \\
Granodiorite & 135.42 & 2.91 & \\
Granodiorite & 129.83 & 3.06 & $\pm 0.6 \%$ \\
Quartzdiorite & 149.86 & 2.56 & \\
Quartzdiorite & 151.60 & 2.53 & $\pm 0.5 \%$ \\
Marble & 87.70 & 5.27 & \\
Marble & 86.79 & 5.32 & \\
\hline \hline
\end{tabular}

maximum temperature (TMAX). This is very convenient because with only one reading the thermal conductivity can be inferred. In this way, the parameter needed to infer thermal conductivity (TMAX) can be obtained directly, saving measuring time and making unnecessary the use of data storage capacity. Obviously, the pulse duration has to fall within region E. According to our experience a heat pulse of about $170 \mathrm{~s}$ is adequate for measuring the thermal conductivity range of consolidated rocks.

\section{CALIBRATHON AND RESULTS}

The measuring design presented here does not provide absolute measurements. Calibration to convert the measured parameters into thermal conductivity is needed. A. calibration has been carried out using ten rock samples which have been chosen such that cover a wide spectrum of thermal conductivities, and their fabrics and composition are as homogeneous as possible in each of them. From each sample three disks of different thickness were cut to be measured with a divided-bar apparatus. Furthermore, a parallelepiped piece of the same sample was cut to be measured with our instrument. Each measure has been performed three times.

The measuring conditions for our instrument are the following: (a) The initial temperature conditions have to be $T_{m}=T_{c}=T_{a}$, where $T_{m}$ is the sample temperature, $T_{c}$ is the heater temperature, and $T_{a}$ is the ambient temperature. We have considered the thermal equilibrium to be reached when the voltmeter reading remains constant for five readings which implies a sensor temperature variation of less

TABLE III. Reproducibility of the conductometer in the continuous heat source mode.

\begin{tabular}{lccc}
\hline \hline Sample & $\begin{array}{c}\text { SLOPE } \\
\left(\mu \mathrm{V}^{-1} \times 10^{2}\right)\end{array}$ & $\begin{array}{c}\text { Conductivity } \\
\left(\mathrm{W} \mathrm{m}^{-1} \mathrm{~K}^{-1}\right)\end{array}$ & Reproducibility \\
\hline Granodiorite & 16.31 & 2.78 & \\
Granodiorite & 16.29 & 2.78 & $\pm 2.8 \%$ \\
Granodionite & 17.11 & 2.94 & \\
Grainstone & 17.61 & 3.04 & $\pm 0.8 \%$ \\
Grainstone & 17.25 & 2.99 & \\
Granite & 20.91 & 3.76 & $\pm 3.0 \%$ \\
Granite & 19.90 & 3.54 & \\
\hline
\end{tabular}

Thermal conductivity 
TABLE IV. Thermal conductivities obtained with divided-bar, heat pulse source, and continuous heat source mode.

\begin{tabular}{|c|c|c|c|c|c|}
\hline Sample & $\begin{array}{l}K \text { (reference) } \\
\left(\mathrm{W} \mathrm{m}^{-1} \mathrm{~K}^{-1}\right)\end{array}$ & $\begin{array}{c}K(\text { TMAX) } \\
\left(\mathrm{W} \mathrm{m}^{-1} \mathrm{~K}^{-1}\right)\end{array}$ & $\begin{array}{l}\text { Error } \\
(\%)\end{array}$ & $\begin{array}{c}K(\text { SLOPE) } \\
\left(\mathrm{W} \mathrm{m}^{-1} \mathrm{~K}^{-1}\right)\end{array}$ & $\begin{array}{l}\text { Error } \\
(\%)\end{array}$ \\
\hline Massive gypsum & $1.44 \pm 0.18$ & 1.45 & 0.70 & 1.44 & 0.00 \\
\hline Basalt & $2.22 \pm 0.16$ & 2.15 & 3.15 & 2.27 & 2.25 \\
\hline Quartzdionite & $2.54 \pm 0.03$ & 2.53 & 0.40 & 2.43 & 4.33 \\
\hline Granodiorite & $2.86 \pm 0.17$ & 2.91 & 1.75 & 2.78 & 2.80 \\
\hline Grainstone & $2.92 \pm 0.03$ & 2.97 & 1.71 & 3.04 & 4.11 \\
\hline Granite & $3.28 \pm 0.03$ & 3.79 & 15.55 & 3.76 & 14.63 \\
\hline Dolomite & $3.34 \pm 0.15$ & 3.37 & 0.90 & 3.38 & 1.20 \\
\hline Sandstone & $4.39 \pm 0.28$ & 3.88 & 11.61 & 3.58 & 18.45 \\
\hline Microconglomerate & $4.49 \pm 0.33$ & 4.34 & 3.34 & 4.44 & 1.12 \\
\hline Marble & $5.19 \pm 0.07$ & 5.27 & 1.54 & 5.21 & 0.40 \\
\hline
\end{tabular}

than $4 \times 10^{-5} \mathrm{~K} \mathrm{~s}^{-1}$. (b) In heat-pulse-source mode the power dissipated at the heat device is $0.612 \mathrm{~W}$. In continuous heat source mode the value is $1.875 \mathrm{~W}$. (c) A reading is taken every second. The time required to measure is $170 \mathrm{~s}$ in heat-pulse-source mode, whereas in continuous heat source mode $700 \mathrm{~s}$ are needed to infer the inflection point.

Table I allows us to compare the results obtained using the divided bar and our conductometer operating in pulse and continuous modes.

A third-degree polynomial has been adjusted between divided-bar absolute values and the parameters (TMAX, SLOPE) obtained with the conductometer. Eight out of ten results have been used because of the anomalous values obtained for samples of granite and sandstone. These anomalous values are due to differences in fabrics and composition between the samples measured with the divided bar and our instrument, in spite of the cautions taken in the sample preparation. In addition, the two anomalous samples and three more were measured by courtesy of the ETH-Zurich with a QTM-2 (Showa-Denko) apparatus which gave results similar to those obtained in our laboratory.

The polynomials obtained are (Figs. 5 and 6 )

$y=-1.52696 x^{3}+24.0182 x^{2}-138.000 x+371.383$,

in heat-pulse-source mode, and

$y=-0.106131 x^{3}+0.640374 x^{2}+3.87698 x+2.85495$, in continuous heat source mode.

An estimation of the reproducibility has been realized by repetitive measurements of a three-sample set (Tables II and III). From Tabies II and III we consider the reproducibility to be better than $2.5 \%$ in heat-pulse-source method and better than $3 \%$ in continuous heat source mode.

Table IV shows a comparison of the results obtained using both modes with the reference conductivities obtained with the divided bar. Except for the granite and sandstone the maximum error is less than $4 \%$ in heat-pulse-source mode and less than $5 \%$ for continuous heat source mode.

From the obtained results we favor the use of the heatpulse-source method because it has been shown to slightly improve the precision and reproducibility of the measurements as compared with the continuous heat source method.

\section{ACKNOWLEDGRENTS}

The authors are indebted to G. Vasseur (Montpellier) for helpful advice on instrument design and to L. Rybach and J. C. Griesser for QTM measurements at the ETH-Zurich. J. Palau and X. Berastegui (Servei Geologic, Barcelona) kindly instructed us on a representative choice of rock samples. We thank LL. Sole (C. S. I. C., Barcelona) for allowing us to use their saw to prepare the cuttings. This work has been partially supported by the "Fundacio Bosch \& Gimpera."

'R. Von Herzen and A. E. Maxwell, J. Geophys. Res. 64, 1557 (1959).

${ }^{2}$ A. P. Shushpanov and P. I. Shushpanov, Inzh. Fiz. Zh. 3, 74 (1960).

${ }^{3}$ C. R. B. Lister, J. R. Astron. Soc. 57, 451 (1979).

${ }^{4} J$. V. Beck and S. All Araju, J. Heat Transfer 96, 60 (1974).

${ }^{5}$ S. Gasharov and P. Petrov, Tectonophysics 103, 315 (1984).

'J. H. Sass, C. Stone, and R. J. Munroe, J. Volcanol. Geother. Res. 20, 145 (1984).

${ }^{7}$ H. S. Carslaw and J. C. Jaeger, Conduction of Heat in Solids, 2nd ed. (Oxford University, Oxford, 1959). 